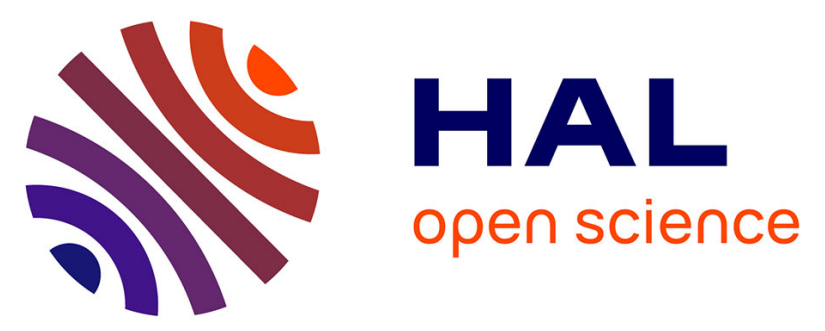

\title{
Comparison between oral and intravenous fludarabine plus cyclophosphamide regime as front-line therapy in patients affected by chronic lymphocytic leukaemia: influence of biological parameters on the clinical outcome
}

Luca Laurenti, Laura Padua, Michela Tarnani, Nicola Piccirillo, Paolo

Falcucci, Giovanni d'Arena, Idanna Innocenti, Sara Marietti, Dimitar G. Efremov, Patrizia Chiusolo, et al.

\section{- To cite this version:}

Luca Laurenti, Laura Padua, Michela Tarnani, Nicola Piccirillo, Paolo Falcucci, et al.. Comparison between oral and intravenous fludarabine plus cyclophosphamide regime as front-line therapy in patients affected by chronic lymphocytic leukaemia: influence of biological parameters on the clinical outcome. Annals of Hematology, 2010, 90 (1), pp.59-65. 10.1007/s00277-010-1025-y · hal-00555319

\author{
HAL Id: hal-00555319 \\ https://hal.science/hal-00555319
}

Submitted on 13 Jan 2011

HAL is a multi-disciplinary open access archive for the deposit and dissemination of scientific research documents, whether they are published or not. The documents may come from teaching and research institutions in France or abroad, or from public or private research centers.
L'archive ouverte pluridisciplinaire HAL, est destinée au dépôt et à la diffusion de documents scientifiques de niveau recherche, publiés ou non, émanant des établissements d'enseignement et de recherche français ou étrangers, des laboratoires publics ou privés. 


\section{Editorial Manager(tm) for Annals of Hematology \\ Manuscript Draft}

\section{Manuscript Number: AOHE-D-10-00105R2}

Title: Comparison between oral and intravenous fludarabine plus cyclophosphamide regime as frontline therapy in patients affected by chronic lymphocytic leukaemia: influence of biological parameters on the clinical outcome.

Article Type: Original Article

Keywords: Keywords: Chronic Lymphocytic Leukemia, Fludarabine, Cyclophosphamide, biological prognostic factors.

Corresponding Author: MD luca laurenti,

Corresponding Author's Institution:

First Author: Luca Laurenti

Order of Authors: Luca Laurenti; luca laurenti; Laura De Padua; Michela Tarnani; Nicola Piccirillo; Paolo Falcucci; Giovanni D'Arena; Idanna Innocenti; Sara Marietti; Dimitar G Efremov; Patrizia Chiusolo; Gina Zini; Federica Sorà; Simona Sica; Giuseppe Leone

Abstract: The fludarabine plus cyclophosphamide (FC) regimen was reported to be superior to chlorambucil or fludarabine alone in terms of complete response, overall response and progression free survival in previously untreated patients with chronic lymphocytic leukemia (CLL). In the present study, we compared the efficacy and toxicity of FC administered through oral and intraveneous route in 65 untreated patients affected by advanced CLL. No statistical differences were noticed between the two routes of administration in terms of overall response (OR), progression free survival (PFS), time to retreatment (TTR) and overall survival (OS) of analyzed patients. We also assessed the influence on the clinical outcome of the mutation status of the immunoglobulin variable region heavy chain (IgVH) gene, of cytogenetic abnormalities and of the expression of ZAP70 and CD38 in patients primary samples. Among the 58 evaluable patients, 31 (53\%) achieved a complete response and 18 (31\%) a partial response. The median PFS was 35 months, median TTR was 42 months and median OS was not reached .after 45 months (range 1-161).

A significantly lower OR rate was noticed in patients with high-risk cytogenetic abnormalities (del 17p, del 11q).In this study, high-risk cytogenetic abnormalities and unmutated IgVH genes were independent predictors of TTR.

These results underline the importance of biological stratifications in front-line treatment of CLL patients. We confirm that FC is an effective regimen, with mild toxicities; it could be recommended for patients with low risk biological parameters, who represent in our experience about $30 \%$ of the total.

Response to Reviewers: 
Comparison between oral and intravenous fludarabine plus cyclophosphamide regime as front-line therapy in patients affected by chronic lymphocytic leukaemia: influence of biological parameters on the clinical outcome.

\section{Corresponding Author:}

Luca Laurenti, MD

Department of Haematology

Catholic University Hospital "A. Gemelli",

Rome, Italy.

Largo A. Gemelli, 8

00168 Roma-Italia

Phone: 39-06-35503953

Fax: 39-06-3017319

e-mail: 1.laurenti@rm.unicatt.it

Keywords: Chronic Lymphocytic Leukemia, Fludarabine, Cyclophosphamide, biological prognostic factors. 


\section{Abstract}

The fludarabine plus cyclophosphamide (FC) regimen was reported to be superior to chlorambucil or

fludarabine alone in terms of complete response, overall response and progression free survival in previously untreated patients with chronic lymphocytic leukemia (CLL). In the present study, we compared the efficacy and toxicity of FC administered through oral and intraveneous route in 65 untreated patients affected by advanced CLL. No statistical differences were noticed between the two routes of administration in terms of overall response (OR), progression free survival (PFS), time to retreatment (TTR) and overall survival (OS) of analyzed patients. We also assessed the influence on the clinical outcome of the mutation status of the immunoglobulin variable region heavy chain $(\mathrm{IgVH})$ gene, of cytogenetic abnormalities and of the expression of ZAP70 and CD38 in patients primary samples. Among the 58 evaluable patients, $31(53 \%)$ achieved a complete response and $18(31 \%)$ a partial response. The median PFS was 35 months, median TTR was 42 months and median OS was not reached .after 45 months (range 1-161).

A significantly lower OR rate was noticed in patients with high-risk cytogenetic abnormalities (del 17p, del 11q).In this study, high-risk cytogenetic abnormalities and unmutated $\operatorname{IgVH}$ genes were independent predictors of TTR.

These results underline the importance of biological stratifications in front-line treatment of CLL patients. We confirm that FC is an effective regimen, with mild toxicities; it could be recommended for patients with low risk biological parameters, who represent in our experience about $30 \%$ of the total. 


\section{Introduction}

The purine analogue fludarabine inhibits the repair of DNA interstrand crosslinks caused by alkylating

agents [1,2]. The synergistic effect between fludarabine and cyclophosphamide prompted investigation of a combined regimen of the two drugs for the treatment of patients affected by CLL. Several studies, including two randomized trials, demonstrated that combined administration of fludarabine and cyclophosphamide (FC) is superior to other standard treatments for CLL, leading to the wide acceptance of this protocol as front-line therapy in patients with advanced disease [3-6]. More recently, a randomized controlled trial comparing FC versus FC plus Rituximab (FCR) showed an improved overall response (OR) rate, progression-free survival (PFS) and overall survival (OS) in patients receiving immunochemotherapy, and FCR can be considered the new standard for first-line treatment of physically fit CLL patients [7]. Moreover, the same trial showed that the effect of FCR vs FC in terms of OR rate is not improved in patients with mutated IgVH and standard risk FISH, suggesting that biological risk stratification may be necessary to determine the optimal first-line treatment in individual patients [8]. FCR also significantly improved PFS in previously treated CLL. [9]

We conducted a multicentre retrospective study to detect the efficacy and safety of FC as front-line therapy of CLL, comparing two different schedules of treatment. In an effort to understand the impact of the molecular prognostic features on response and outcome, we studied the influence of $\operatorname{IgVH}$ mutation status, interphase cytogenetic abnormalities, and expression of ZAP-70 and CD38 on the overall response rate, progression free survival, time to re-treatment (TTR), and overall survival. 


\section{Materials and Methods}

\section{Patients and Treatment}

This multicentre retrospective study included previously untreated patients with progressive CLL defined according to National Cancer Institute (NCI) criteria [10,11]. Patients with previous episodes of autoimmune haemolytic anaemia or positive direct/indirect Coombs test were excluded. The study was approved by the internal Ethical Committee at our Institution and all patients provided written informed consent.

Biological status of enrolled patients was tested at diagnosis or before starting treatment in all cases. Thirty eight patients received oral fludarabine $\left(30 \mathrm{mg} / \mathrm{m}^{2}\right)$ and oral cyclophosphamide $\left(250 \mathrm{mg} / \mathrm{m}^{2}\right)$ and 27 patients received intravenous fludarabine $\left(25 \mathrm{mg} / \mathrm{m}^{2}\right)$ and intravenous cyclophosphamide (250 $\mathrm{mg} / \mathrm{m}^{2}$ ); both treatments were administered for three consecutive days every 4 weeks for six cycles.

Patients receiving the oral formulation (os) or the intravenous administration (iv) were not hospitalized.

Patients receiving the oral or intravenous route in this study were randomly chosen. A slight reduction of the total dose of oral FC was adopted according to previous data from our institution [12].

Prophylaxis against herpes zoster and pneumocystis carinii was provided by administration of acyclovir $800 \mathrm{mg}$ tid, and trimetoprim-sulfametoxasol $960 \mathrm{mg}$ bid for 2 consecutive days every week, during the entire treatment period and 2 months after. Administration of G-CSF was used for grade IV neutropenia independently from the onset of fever. Treatment was delayed for up to two weeks in case of grade III-IV cytopenia, at day 28 of each cycle. Once the cytopenia was reduced to grade I or II, treatment was reinstituted with a $25 \%$ dose reduction of both drugs for all subsequent cycles. A further $25 \%$ dose reduction was performed in case of grade III-IV cytopenia in subsequent cycles. Treatment was discontinued in case of progressive disease, autoimmune haemolytic anemia, autoimmune thrombocytopenia or pure red cell aplasia (PRCA) .

\section{Study End Points}

The efficiency of the treatment was assessed according to the NCI-WG response criteria [10, 11]. Progression-free survival, time to re-treatment and overall survival were defined from the date when therapy was started to the date of progression, re-treatment and death or last follow-up, respectively. 
The primary end-point of the study was the response rate and safety of oral and intravenous treatment with fludarabine and cyclophosphamide. The secondary end-point was an evaluation of the influence of biological parameters on OR rate, PFS, TTR and OS.

\section{Fluorescent In Situ Hybridization}

Interphase FISH studies were performed according to the manufacturer's instructions on peripheral blood samples using a set of four commercial probes (Abbott, Wiesbaden-Delkenheim, Germany) [13]. An alpha satellite DNA probe CEP12 directly labelled with SpectrumOrange was used to detect aneuploidy of chromosome 12. LSI p53 together with CEP17 alpha satellite DNA probes labelled with SpectrumOrange and SpectrumGreen, respectively, were used to evaluate chromosome deletion at 17p13.1. LSI RB and LSI ATM, both labelled with SpectrumOrange, were used to detect chromosome deletion at 13q34 and 11q22-23, respectively. Prior to hybridization, the slides were dehydrated with three ethanol washes $(70 \%, 85 \%$, and $100 \%)$ and air-dried. A 10 microL probe mixture was added to each slide, and the slides were covered with a coverslip. The slides were then denatured using a Vysis Hybrite set (Vysis Inc.) at a melting temperature of $75^{\circ} \mathrm{C}$ for 5 minutes and then hybridized at $37^{\circ} \mathrm{C}$ overnight. The coverslips were removed, and the slides were washed for 2 minutes in $0.4 \mathrm{x} \mathrm{SSC}$ at $73^{\circ} \mathrm{C}$ and then in $2 \times \mathrm{SSC}$ at room temperature for 2 minutes. Nuclei were counterstained with 46-diamindino-2-phenylindole dihydrochloride and cover again with a coverslip. A minimum of two hundred consecutive, qualifying, interphase nuclei were analysed using a Zeiss AxiosKop 2 plus fluorescent microscope. The normal cut-off value was established by analysing 10 peripheral blood samples from normal blood subjects.

\section{IgVH gene mutational analysis}

Peripheral blood mononuclear cells (PBMCs) were separated by Ficoll gradient centrifugation (Amersham Biosciences, Uppsala, Sweden). Total cellular RNA was isolated from PBMCs using the Trizol reagent (Invitrogen) according to the manufacturer's instructions. RNA was reverse transcribed using random hexamers and then amplified with the polymerase chain reaction (PCR) using a degenerate $\mathrm{VH}$ framework 1 (FWR1) primer in combination with $\mathrm{C} \square, \mathrm{C} \square$ and $\mathrm{C} \square$ reverse primers. Samples that failed to amplify with these combinations were amplified with a mixture of forward primers complementary to leader sequences of VH family 1 to 6 . PCR products were purified with the QIAquick PCR purification kit (Qiagen, Valencia, CA) and either sequenced directly or cloned with 
the PCR Cloning Plus kit (Qiagen) [14]. Sequencing analysis was performed with the BigDye Terminator v3.1 Cycle Sequencing kit on an ABI 3100 genetic analyzer (Applied Biosystems, Foster

City, CA). Candidate germ-line genes were assigned by searching the VBASE directory. Sequences with less than $2 \%$ differences from germ-line VH sequences were considered unmutated.

\section{Flow Cytometry}

Fifty microliters of whole blood in EDTA containing approximately $5 \times 10^{5}$ cells were incubated at room temperature in the dark for 20 minutes with CD3 PE-Cy5, CD56 PE-Cy5, CD5 FITC and CD19 PE-Cy7 to identify surface antigens. After incubation, samples were centrifuged at $1500 \mathrm{rpm}$ for 5 minutes. Cell pellets were resuspended in 100 microL of fixation medium A (caltag Laboratories, Burlingame, CA, USA) and incubated at room temperature in the dark for 15 minutes. Then cells were pelleted again at $1500 \mathrm{rpm}$ for 5 minutes, resuspended in 100 microL of permeabilization medium B (Caltag Laboratories) and incubated with the ZAP-70 monoclonal antibody at room temperature in the dark for one hour. The ZAP-70 antibody was R-phycoerythrin conjugated (clone 1E 7.2, Caltag Laboratories). Samples were washed twice by centrifugation at $1500 \mathrm{rpm}$ for 5 minutes and resuspended in 500 microL of phosphate-buffered saline. Finally, $1 \times 10^{4}$ fixed cells were analyzed by flow cytometry (BD FACSCanto, Becton Dickinson) using the gating strategy described by Crespo et al [15]. Cases expressing more than 20\% ZAP-70 positive CLL B-cells were defined as positive.

CD38 analysis was performed in the setting of routine cellular immunophenotyping using PEconjugated anti-CD38 from Becton Dickinson (San Jose, CA). Peripheral blood mononuclear cells, separated from heparinised venous blood by density gradient centrifugation (Histopaque-1077, Sigma Diagnostics, St Louis, MO) were analyzed for surface expression of CD19/CD5/CD38 and CD19/CD5/CD23 by triple colour immunofluorescence. Isotype-matched negative controls were used in all the assays to determine positive from negative results. Flow cytometric analyses were performed on a FACS Calibur flow cytometer (Becton Dickinson Immunocytometry Systems) equipped with argon and red diode lasers. The CellQuest software system (Becton Dickinson Immunocytometry Systems) was used to acquire and to analyze data. Cases expressing more than $30 \% \mathrm{CD} 38$ positive CLL B-cells were defined as positive [16]. 
First we analyzed the impact of the two different routes of administration (oral / iv) on the following outcomes: Overall Response rate defined as achievement of complete response (CR), partial response (PR), and nodular PR; PFS defined as the time interval from the first day of treatment until disease progression; TTR calculated from the first day of treatment until initiation of a new treatment; overall survival time, calculated from the first day of treatment until death from any cause. For nonresponders and progressive disease, date of progression was when no response or progressive disease was recorded.

The following parameters were used: sex, age $(<65$ years vs $>=65$ years $)$, Binet stage $(\mathrm{B} / \mathrm{C})$, cytogenetic abnormalities (high risk, HR vs standard risk SR cytogenetics,), IgVH mutational status, expression of ZAP-70 and CD38. We chose to consider patients with 11q deletion and 17p deletion together as a high-risk cytogenetic group because of the small number of patients with these abnormalities ( 8 patients with del $17 \mathrm{p}$ and 7 patients with del $11 \mathrm{q}$ ). Response to treatment was also included for PFS/TTR/OS outcomes.

The chi-square test was used to analyze the categorical variables. Univariate survival analysis was performed using the Kaplan-Meier method and Logrank test. The Cox proportional hazards model and the logistic regression model were used to test the association of the covariates with outcomes of $\mathrm{PFS} / \mathrm{TTR} / \mathrm{OS}$ and OR rate, respectively. Only variables that in univariate analysis reached a $* \mathrm{P}$ value $<0.25$ were included in the model. The models were built using the Backward Stepwise (Wald) procedure. The results are expressed as Hazard Ratios (HRs) and 95\% confidence intervals (CI95\%). Statistical analysis was performed using the Statistical Package for Social Sciences (SPSS), release 16.0 for Windows. A $* \mathrm{P}$ value less than 0.05 was considered significant. 


\section{Results}

\section{Patient characteristics}

Sixty-five previously not treated patients with progressive CLL from three different Italian institutions were enrolled in the study. Thirty-eight patients received oral schedule treatment at the Catholic University Medical Centre; 27 patients were enrolled from a medical center that had adopted iv schedule of FC combination: 15 patients were treated at the Viterbo Hospital and 12 patients at the San Giovanni Rotondo Hospital. Median time between the diagnosis and the beginning of therapy was 27 months (range 1-137). Forty-eight patients were male and 17 were female, with a median age of 65 years (range, 44 to 78 years). Fifty-two patients were in stage B/II, 4 in stage C/III, and 9 in stage C/IV. Thirty-three patients had not mutated and 18 had mutated IgVH genes; in 14 patients the mutation status was not determined. Fifteen patients $(23 \%)$ had the 'high risk' cytogenetic abnormality del(11q22.3) or del(17p13.1). Twenty eight patients expressed ZAP-70 (cut-off 20\%) and 23 patients were CD38-positive (cut-off 30\%). (Table 1).

\section{Response}

Fifthy eight patients were evaluable for response to treatment. Seven patients did not complete the planned treatment, two of them because of onset of immune thrombocytopenia that developed after 2 and 4 cycles; one because of PRCA that developed after the first cycle and one because of skin toxicity that developed after 2 cycles. The other three patients died during treatment, two from pneumonia that occurred in the setting of grade IV neutropenia after the first and second cycle, respectively, and one from sudden cardiac death after 3 cycles of therapy.

Thirty one patients (53\%) obtained a complete response and $18(31 \%)$ a partial response, with an OR rate of $84 \%$. Four patients showed stable disease and five patients experienced disease progression after 2, 3, 4 (two patients) and 6 cycles respectively. No statistical differences were observed when comparing the OR in the group of patients treated with iv FC $(83 \%$ OR) and those treated with oral FC $(85 \%$ OR).

The high-risk cytogenetic patients group included 8 patients with del 17p and 7 with del 11q. We noticed 4 response to treatment in each cytogenetic alteration, even if 3 of four $17 \mathrm{p}$ deletion patients showed less than $20 \%$ positive cells.

Univariate analysis showed cytogenetic abnormalities as the only factor influencing the overall response rate $(* \mathrm{P}=0,016)$. $\mathrm{CD} 38$ expression and stage of disease were also included in the multivariate 
model because of a $* \mathrm{P}$ value $<0.25$ in the univariate analysis, but only high risk cytogenetic abnormalities were identified as an independent prognostic parameter (HR 0,093; CI 0,015-0,580). The IgVH, ZAP-70 and CD38 categories did not show statistical differences in terms of OR rate (table 2).

\section{PFS, TTR and $O S$}

Median PFS was 35 months (range 2-118). In univariate analysis,, response to treatment was significantly associated with PFS $(* \mathrm{P}<0.001)$. IgVH mutation status $(* \mathrm{P}=0.132)$ and FISH $(* \mathrm{P}=0.058)$ was also included in the multivariate model because of a $* \mathrm{P}$ value $<0.25$, but only response to therapy was identified as an independent prognostic parameter (table2).

Median TTR was 42 months (range 2-118). Response to treatment ( $\left.{ }^{*} \mathrm{P}<0.001\right)$, IgVH mutation status $(* \mathrm{P}=0.026)$, cytogenetic abnormalities $(* \mathrm{P}=0.007)$ and age $(* \mathrm{P}=0.25)$ were investigated in the multivariate model; response to treatment, $\mathrm{IgVH}$ and cytogenetic abnormalities emerged as independent prognostic factors (table 2, figure 1 and 2).

Median OS was not reached after 45 months (range 1-161). During the study period, 19 patients died. In univariate analysis only response to treatment achieved a statistically significant value $(* \mathrm{P}<0.001)$, whereas response to treatment and age emerged as independent prognostic factors from the multivariate analysis (table 2).

\section{Hematological and extra-hematological toxicity}

Twelve patients developed grade III and 21 patients developed grade IV neutropenia (37\% after iv FC and $29 \%$ after oral FC). Two of these patients, both of them from the iv FC arm, died because of pneumonia after the first and the second cycle of therapy. Three patients developed grade III or grade IV anemia that required red blood cell transfusions. Two patients developed immune thrombocytopenia after the 2 nd and 4 th cycle and one patient developed pure red cell aplasia after the 1st cycle, which regressed after treatment with steroids and Rituximab.

As for extra-hematological toxicity, nausea was reported by 18 patients and 8 of them also experienced vomiting; as expected these complications were predominantly observed in the oral FC group. One patient developed fever of unknown origin after the first cycle of chemotherapy, which spontaneously resolved without hospitalization. One patient showed severe skin toxicity due to chemotherapy and discontinued treatment after 2 cycles. One patient died after 3 cycles of therapy 
from sudden cardiac death due to congenital abnormalities of the coronary arteries discovered during autopsy (table 3).

1

2

3

4

5

6

7

8

9

10

11

12

13

14

15

16

17

18

19

20

21

22

23

24

25

26

27

28

29

30

31

32

33

34

35

36

37

38

39

40

41

42

43

44

45

46

47

48

49

50

51

52

53

54

55

56

57

58

59

60

61 


\section{Discussion}

A large multicenter study recently demonstrated that fludarabine plus cyclophosphamide is superior to

chlorambucil or fludarabine alone as front-line treatment of CLL with respect to complete response, overall response and progression free survival [4]. In addition, FC was found to be superior to chlorambucil or fludarabine alone in all age groups, including patients older than 70 years, suggesting that FC can be considered the standard treatment for chronic lymphocytic leukaemia. The results of this study confirmed our preliminary data and other published studies [3-6,12,17] regarding the efficacy and safety of fludarabine plus cyclophosphamide as front line therapy in patients affected by chronic lymphocytic leukemia (table 4).

Table 4 shows a similar response to therapy among five data series on the FC regimen, even if the dose, timing and route of administration were different in the reported studies. The table also underlines differences in PFS between different studies that could be explained by lower cumulative dose of chemotherapy or different schedule of administration. No comparison of TTR and OS is possible because of unavailability of comparable data.

In our study we did not detected any significant difference in the clinical outcome between the iv FC and the oral FC groups, even though the total dose of fludarabine and cyclophosphamide was higher in the iv FC group. Patients who received oral combination were previously reported in our published series. In that series, we adopted a total dose of FC slightly decreased compared to other published studies in order to reduce the side effects of the drugs and to avoid hospitalization in elderly patients with comorbidities. The higher intravenously dose could explain why patients from the iv FC group experienced more often grade III/IV neutropenia and more severe infections, including two fatal pneumonias. On the other side, nausea and vomiting occurred more often among patients receiving oral FC.

Results from a large non randomized trial of FCR and preliminary results of a randomized, controlled trial comparing FC versus FCR showed an improved response rate and progression free survival in patients who received the immunochemotherapy regimen, so FCR has became the new standard firstline treatment for physically fit CLL patients [7, 18]. The same study reported that the effect of FCR vs FC on the OR rate is not higher in patients with IgVH mutated CLL and standard risk FISH [8]. In the monoclonal antibody era, when the association with chemotherapy shows certain benefits on clinical outcome but also more haematological and extra haematological toxicities, a stratification of the biological risk of patients prior to treatment should be considered. In our series, we found that the 
'high risk' cytogenetic abnormalities are independent predictors of a lower OR rate as well as a shorter TTR. In addition, unmutated IgVH genes are significantly associated with a shorter TTR, whereas expression of CD38 and ZAP-70 has no significant impact on clinical outcome.

As expected, response to treatment was an independent predictor of longer PFS, TTR and OS, while age was predictive of longer OS in our study. Biological risk stratifications demonstrated that patients with "high risk" cytogenetic abnormalities and germ line $\mathrm{IgVH}$ genes have a shorter time to retreatment following FC chemotherapy, suggesting that high risk untreated younger patients affected by progressive CLL should be candidates to receive chemo-immunotherapy. Conversely, a consistent group of low risk CLL patients emerged from the biological stratification. These patients display mutated IgVH and standard risk FISH and they respond to FC with an high OR rate and a prolonged TTR. A good compliance to treatment and the strong tolerability exhibited by this substantial proportion of patients argue that they could benefit from FC alone. FCR can be also employed to treat these patients, but haematological and extra-hematological complications can arise in elderly patients (> 70 aa) as elsewhere reported. 


\section{References}

1. Bellosillo B, Villamor N, Colomer D, Pons G, Montserrat E, Gil J (1999) In vitro evaluation of Fludarabine in combination with Cyclophosphamide and/or Mitoxantrone in B-cell chronic lymphocytic leukemia. Blood 94:2836-2843

2. Koehl U, Li L, Nowak B (1997) Fludarabine and Cyclophosphamide: synergistic cytotoxicity associated with inhibition of interstrand cross-link removal[abstract]. Proc Am Assoc Cancer Res $38: 2: 10 \mathrm{a}$

3. Eichhorst BF, Busch R, Hopfinger G (2006) Fludarabine plus cyclophosphamide versus fludarabine alone in first-line therapy of younger patients with chronic lymphocytic leukemia. Blood 107:885-891

4. Catowsky D, Richards S, Matutes E et al (2007) Assessment of fludarabine plus cyclophosphamide for patients with chronic lymphocytic laeukemia (the LRF CLL4 trial): a randomized controlled trial. Lancet 370: 230-39

5. O'Brien S, Kantarjian HM, Cortes J, Beran M, Koller CA, Giles FJ, et al (2001) Results of the Fludarabine and Cyclophosphamide combination regimen in chronic lymphocytic leukemia. J Clin Oncol 19:1414-20

6. Flinn IW, Neuberg DS, Grever MR, Dewald GW, Bennett JM, Paietta EM, et al (2007) Phase III trial of fludarabine plus ciclophosphamide compared with fludarabine for patients with previously untreated chronic lymphocytic leukemia: US Intergroup Trial E2997. J Clin Oncol 25(7):793-8

7. Hallek M, Fingerle-Rowson G, Fink AM et al (2009) First-line treatment with fludarabine (F), cyclophosphamide (C), and rituximab (R) (FCR) improves overall survival in previously untreated patients with advanced chronic lymhocytic leukemia (CLL): results of a randomized phase III trial on behalf of an international group of investigators and the german CLL study group. Blood 114 (22), abs 535

8. Slilgenbauer S, Zenz T, Winkler D et al (2008) Genomic aberrations, VH mutational status, and outcome after fludarabine and cyclophosphamide (FC) or FC plus rituximab (FCR) in the CLL8 trial. Blood 112 (11), abs 781

9. Robak T, Dmoszynska A, Solal-Céligny P et al (2010). Rituximab plus fludarabine and cyclophosphamide prolongs progression-free survival compared with fludarabine and cyclophosphamide alone in previously treated chronic lymphocytic leukemia. J Clin Oncol. 2010 Apr $1 ; 28(10): 1756-65$.

10. Cheson BD, Bennet JM, Grever M, Kay N, Keating MJ, O'Brien S, et al (1996) National Cancer Institute-sponsored Working Group guidelines for chronic lymphocytic leukemia: revised guidelines for diagnosis and treatment. Blood Jun 15; 87(12):4990-7

11. Hallek M, Cheson B.D, Catovsky D, et al (2008) Guidelines for the diagnosis and treatment of chronic lymphocytic leucemia: a report from the International Workshop on Chronic Lymphocytic Leukemia updating the National Cancer Institute-Working Group 1996 guidelines. Blood 111(12):5446-56

12. Laurenti L, Tarnani M, De Padua L. et al (2008) Oral fludarabine and cyclophosphamide as front-line chemotherapy in patients with chronic lymphocytic leukemia. The impact of biological parameters in the response duration. Ann Hematol 87:891-898

13. Dohner H, Stilgenbauer S, Benner A et al (2000). Genomic aberration and survival in Chronic Lymphocytic Leukemia. N Engl J Med 2000; 343: 1910-6

14. Ivanovski M, Silvestri F, Pozzato G et al (1998). Somatic hypermutation, clonal diversity, and preferential expression of the VH 51p1/VL kv325 immunoglobulin gene combination in hepatitis C virus-associated immunocytomas. Blood 1998;91:2433-42.

15. Crespo M, Bosh F, Villamor N, Bellosillo B, Colomer D, Rozman (2003) ZAP-70 expression as a surrogate for immunoglobuline variable region mutations in chronic lymphocytic leukemia. N Engl J Med; 348(18):1764-75 
16. Damle R.N, Wasil T, Fais F, et al (1999). Ig V Gene Mutation Status and CD38 Expression As Novel Prognostic Indicators in Chronic Lymphocytic Leukemia. Blood 94, p1840-1847

17. Cazin B, Divine M, Lepretre S, Travade P, et al (2008) High efficacy with five days schedule of oral fludarabine phosphate and cyclophosphamide in patients with previously untreated chronic lymphocytic leukaemia. British Journal Hematol 143:54-59

18. Tam C.S, O’Brien S, Wierda W, et al (2008) Long-term results of the fludarabine, cyclophosphamide, and rituximab regimen as initial therapy of chronic lymphocytic leukemia. Blood 112:975-980 
${ }^{*}$ Conflict of interest
Click here to download Conflict of interest: Cl.doc

Click here to download Conflict of interest: Cl.doc

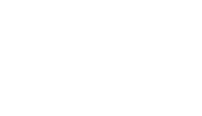

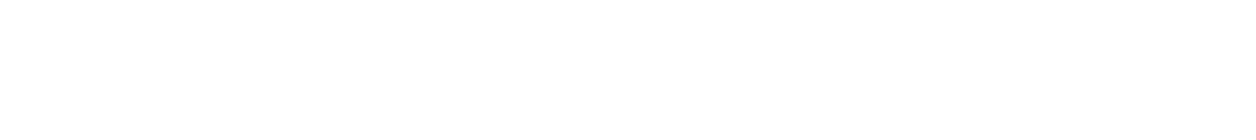
(1) (1) (1) (1) (1) (1) . . .

) 
Figure 1

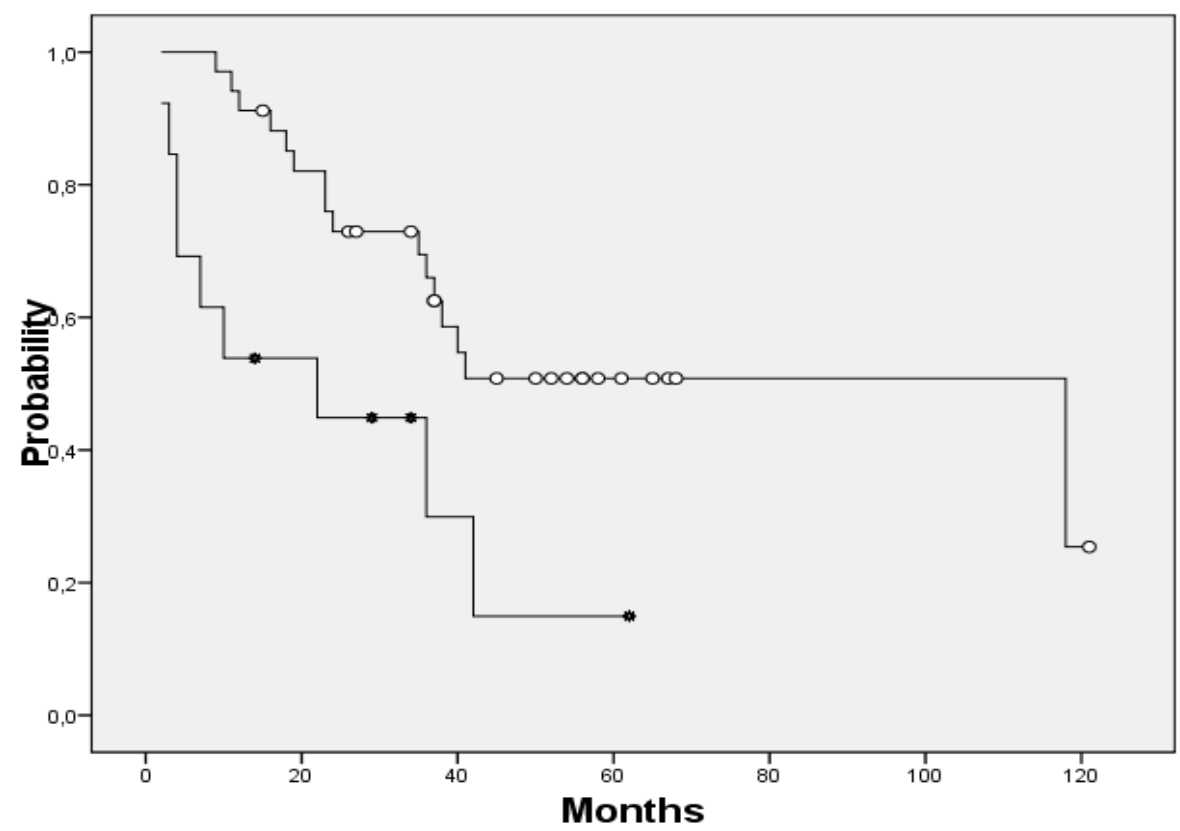


Figure 2

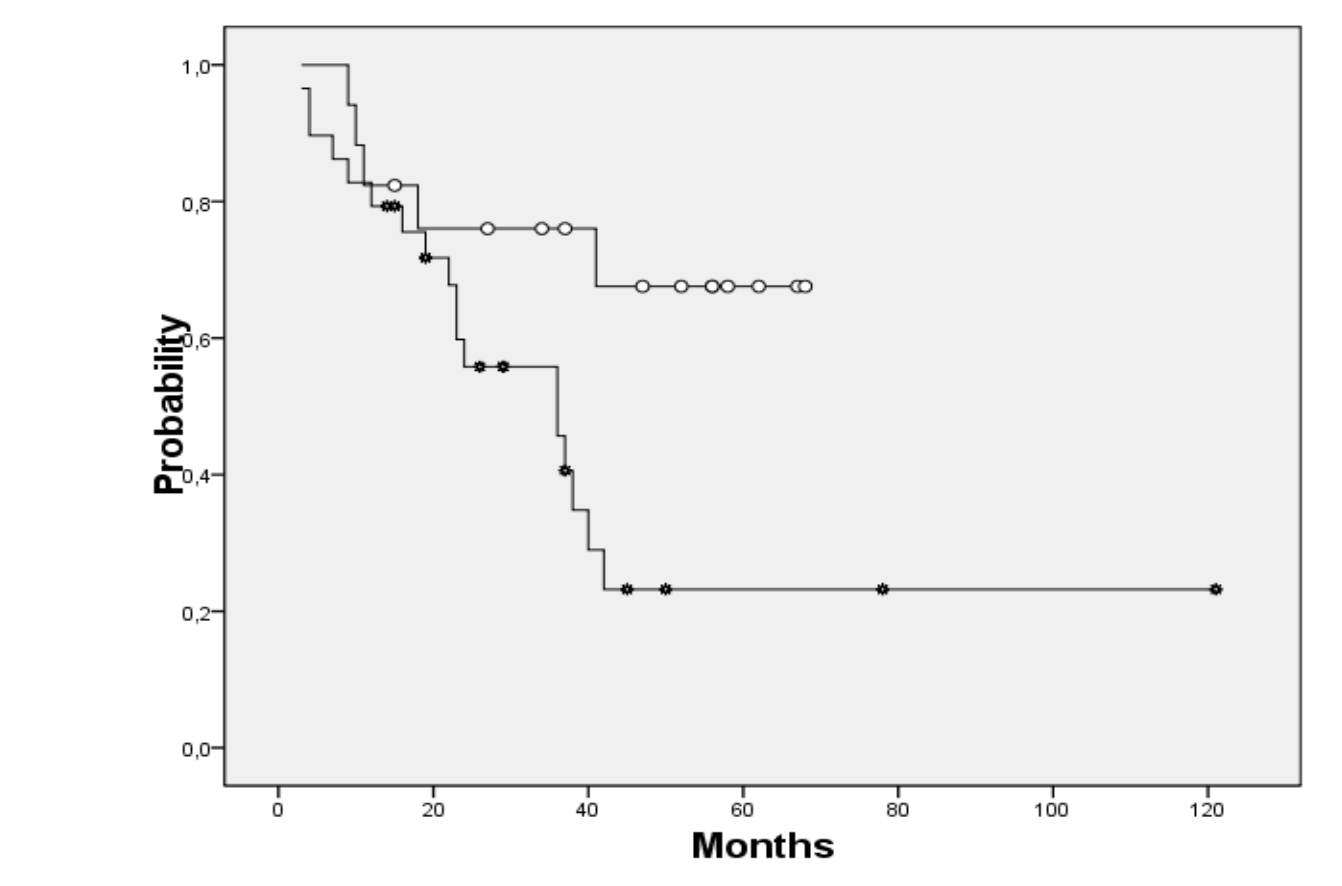

Figure 2

Months

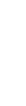



Figure Legend
Click here to download Supplementary Material: figure legends.doc Figure Legend
Click here to download Supplementary Material: figure legends.doc

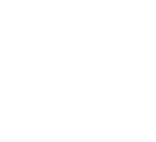

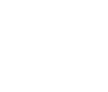

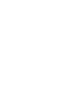

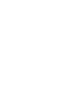
.

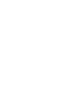

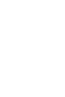

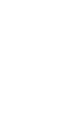

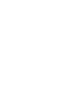

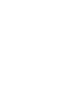

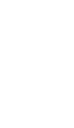

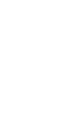
. res

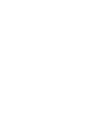

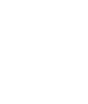

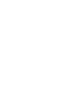

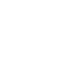

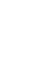

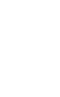

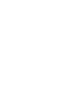

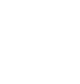
. 10 -

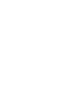

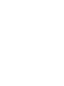

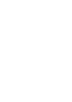

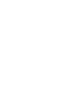

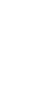
. . . 
Table 2 Multivariate analysis

\begin{tabular}{|c|c|c|c|c|c|c|c|c|c|c|c|c|}
\hline & $H R$ & $\begin{array}{c}\text { OR } \\
\text { CI95\% }\end{array}$ & $P^{*}$ & $H R$ & $\begin{array}{l}\text { TTP } \\
\text { CI95\% }\end{array}$ & $P^{*}$ & $H R$ & $\begin{array}{l}\text { TTT } \\
\text { CI95\% }\end{array}$ & $P^{*}$ & $H R$ & $\begin{array}{c}\text { OS } \\
\text { CI95\% }\end{array}$ & $P^{*}$ \\
\hline $\begin{aligned} \text { Age: } & >65 \text { vs } \\
& <65 \text { years }\end{aligned}$ & & Not included & & & Not included & & & Not included & & 3,656 & $1,046-12,780$ & $0,042^{*}$ \\
\hline $\begin{array}{r}\text { Overall } \mathrm{R} \\
\text { vs } \mathrm{Nr}\end{array}$ & & Not applicable & & 0,102 & $0,035-0,293$ & $<0,001^{*}$ & 0,080 & $0,026-0,248$ & $<0,001^{*}$ & 0,071 & $0,021-0,239$ & $<0,001^{*}$ \\
\hline $\begin{array}{l}\text { FISH: High vs } \\
\text { standard risk }\end{array}$ & 0,093 & $0,015 / 0,580$ & $0,011 *$ & 2,250 & $0,929-5,440$ & $0,072 *$ & 2,587 & $1,024-6,540$ & $0,045^{*}$ & & Not included & \\
\hline $\begin{array}{r}\text { IgVH: NM } \\
\text { vs M }\end{array}$ & & Not included & & 2,195 & $0,860-5,599$ & $0,1^{*}$ & 3,512 & $1,164-10,598$ & $0,026^{*}$ & & Not included & \\
\hline $\begin{array}{l}\text { CD38: pos } \\
\text { vs neg }\end{array}$ & 7,767 & $0.694-86,896$ & $0.096^{*}$ & & Not included & & & Not included & & & Not included & \\
\hline $\begin{array}{l}\text { ZAP-70: pos } \\
\text { vs neg }\end{array}$ & & Not included & & & Not included & & & Not included & & & Not included & \\
\hline
\end{tabular}

Legend: $\mathrm{R}=$ response; $\mathrm{Nr}=$ not response 
Table 1 Clinical and prognostic characteristics of CLL patients

\begin{tabular}{|cccc|}
\hline Features & $\begin{array}{c}\text { Number of } \\
\text { patients }\end{array}$ & $\begin{array}{c}\text { OS FC } \\
\text { (38 patients })\end{array}$ & $\begin{array}{c}\text { IV FC } \\
(27 \text { patients })\end{array}$ \\
\hline Males & 48 & 26 & 22 \\
\hline Females & 17 & 12 & 5 \\
\hline Stage BII & 52 & 33 & 19 \\
\hline Stage CIII/IV & $4 / 9$ & $1 / 4$ & $3 / 5$ \\
\hline FISH HR & 15 & 7 & 8 \\
\hline FISH SR & 36 & 27 & 9 \\
\hline IgVH NM & 33 & 18 & 15 \\
\hline IgVH M & 18 & 14 & 4 \\
\hline CD38 > $30 \%$ & 23 & 11 & 12 \\
\hline CD38 <30\% & 37 & 27 & 10 \\
\hline ZAP70 >=20\% & 28 & 18 & 10 \\
\hline ZAP70 <20\% & 28 & 19 & 9 \\
\hline
\end{tabular}


Table 3 Treatment related toxicity according to NCI

\begin{tabular}{|lcc|}
\hline Kind of toxicity & \multicolumn{1}{c|}{ Number of patients } \\
\hline Hematological (grade III/IV) & OS FC (38 patients) & IV FC (27 patients) \\
Anemia & $2 / 1$ & 0 \\
Neutropenia * & $7 / 11$ & 0 \\
Immune thrombocytopenia & 2 & 1 \\
PRCA & 0 & 4 \\
Extra-hematological & 14 & 1 \\
Nausea & 7 & 2 (exitus) \\
Vomiting & 0 & 0 \\
Pneumoniae* (in grade IV neutropenia) death & 1 (exitus) & 0 \\
Sudden cardiac death & 1 & 0 \\
Skin toxity & 1 & \\
Febrile unknown neutropenia & & 0 \\
\hline
\end{tabular}

$*: \mathrm{p}=\mathrm{ns}$ 
Table 4: Comparison of our experience and published data of FC front-line treatment

\begin{tabular}{|c|c|c|c|c|c|}
\hline & $\begin{array}{c}\text { Laurenti } \\
\text { (65 patients)* }\end{array}$ & $\begin{array}{c}\text { Cazin [17] } \\
\text { (75 patients) }\end{array}$ & $\begin{array}{l}\text { Catovsky [4] } \\
\text { (196 patients) }\end{array}$ & $\begin{array}{c}\text { Flinn [6] } \\
83 \text { patients }\end{array}$ & $\begin{array}{l}\text { Eichhorst [3] } \\
180 \text { patients }\end{array}$ \\
\hline Treatment schedule & os FC/iv FC & os FC & os FC/iv FC & iv FC & iv FC \\
\hline $\begin{array}{l}\mathrm{Mg} / \mathrm{m}^{2} \text { for cycle of } \\
\text { fludarabine }\end{array}$ & $90 / 75$ & 150 & $120 / 75$ & 100 & 90 \\
\hline $\begin{array}{l}\mathrm{Mg} / \mathrm{m}^{2} \text { for cycle of } \\
\text { ciclophosphamide }\end{array}$ & $750 / 750$ & 1000 & $750 / 750$ & 600 & 750 \\
\hline OR rate (CR/PR) & $84(53 / 31)$ & $80(53 / 27)$ & $94(38 / 57)$ & $74(23 / 51)$ & $94(16 / 78)$ \\
\hline $\begin{array}{c}\text { Median (months) } \\
\text { PFS/TTR/OS }\end{array}$ & $35 / 42 / \mathrm{nr}$ at 45 months & $60 / \mathrm{nr} / \mathrm{nr}$ at $7 \mathrm{y}$ & $43 / \mathrm{nd} . / \mathrm{nr}$ at $5 \mathrm{y}$ & $31,6 / \mathrm{nd} / \mathrm{nd}$ & $48 / n d / n d$ \\
\hline
\end{tabular}

Legend: $\mathrm{nr}=$ not reached $\mathrm{nd}=$ not done

* unpublished data 
Editor in chief

We re-submit to your attention the article entitled 'Comparison between oral and intravenous fludarabine plus cyclophosphamide regime as front-line therapy in patients affected by chronic lymphocytic leukaemia: influence of biological parameters on the clinical outcome'.

\section{According to the Reviewer 2 :}

1) The study is retrospective as reported in Methods section.

2) We added the explanation of the route choice in Methods section

3) The use of FCR regimen front line or in relapsed patients was more stressed in the Introduction section.

Detailed comments:

1 Reference n.7 was replaced as suggested

2 The results from REACH study were mentioned in Introduction section, even if a comparison between FC versus FCR in previously treated patients was not discussed because it was not the topic of the paper

3,4,5 We modified table 4 as requested (references, we removed reference 12 and specified 'unpublished' for our data)

\section{Reviewer 3}

1) We clarify in Method section and in discussion the choice of lower dose of oral FC.

2) We added references for the methods used for FISH, IgVH and CD38.

3) Informed consent was just specified in Methods section

4) We added in the last part of the discussion the value of FCR schedula even in low risk patients.

To note that our patients are more elder than CLL8 patients and also at the ASH meeting 2009 Hallek showed more toxicity in terms of neutropenia (III-IV) and infections in elderly (> 70 aa) patients. 


\section{Reviewer 4}

1) As suggested we added the explanation of the route choice in Methods section and we also reported the differences between these two groups of patients in table 1.

2) We added in Method section (statistical analysis) why del 11q and del 17p were both considered as high risk cytogenetics. Also we reported in Results the same percent of response in each subgroup (del 11q and 17p), even if 3 of four patients with del 17p showed a percentage of positive cells less than $20 \%$.

3-4) Biological state of enrolled patients was tested at diagnosis or before starting of treatment in all patients depending on the time of diagnosis. No patients were studied after treatment. We added in Method sections.

5) We did not find statistical differences in the quantities of infectious $(\mathrm{p}=0,088)$ and neutropenia $(\mathrm{p}=0,49)$ between peroral and intravenous administration; we also added in table 3 statistical results.

6) We reported in Results section the median time between diagnosis and therapy; moreover as reported in point 3-4) we stressed that biological study was done at diagnosis or before the treatment. We think that acquired cytogenetic alterations (IgVH and ZAP70 were immutable during the time), according to the literature, don't develop without prior therapy; anyway no patients were studied after starting treatment.

Minor points were changed as requested

We hope it will be re-considered for publication in your journal.

Thanking you in advance for your attention.

Sincerely yours,

Luca Laurenti, MD

Divisione di Ematologia Istituto di Ematologia Universita' Cattolica Sacro Cuore

Largo A. Gemelli, 8 00168 Roma-Italia Phone: 39-06-35503953

Fax: 39-06-3017319 e-mail: 1.laurenti@rm.unicatt.it 